\title{
Land Use Change in Hongta District in Yuxi City, China Based on Archived Landsat Data of the Past 30 Years
}

\author{
Shanyu Zhou, Chaonan Ji, Ran Kang, Hermann Kaufmann \\ Navigation and Remote Sensing Group, Institute of Space Science, Shandong University, Weihai, China \\ Email: Alice_chou@foxmail.com
}

How to cite this paper: Zhou, S.Y., Ji, C.N., Kang, R. and Kaufmann, H. (2018) Land Use Change in Hongta District in Yuxi City, China Based on Archived Landsat Data of the Past 30 Years. Journal of Computer and Communications, 6, 138-145. https://doi.org/10.4236/jcc.2018.611013

Received: October 27, 2018

Accepted: November 12, 2018

Published: November 19, 2018

\begin{abstract}
Urban expansion, which has affected ecosystem functioning and services at local to global scale, is projected to have aggravating impacts on landscape in the future. A detailed study on spatiotemporal patterns of Hongta District in Yuxi City over a relatively long timeframe was conducted using multi-temporal Landsat TM, ETM+ and OLI data of 1987-2017 associated with Geographic Information System techniques and landscape analysis approaches. Results showed that sealed surfaces (urban area) have expanded from $1.4 \%$ to $11.8 \%$, with an annually increasing rate of $7.63 \%$. Trees, farm areas and barren lands all underwent a slight reduction during this time period, whereby $23.8 \%$ of farms and $9.8 \%$ of bare lands transferred into sealed surfaces. Focusing on the urban expansion pattern, it presents a uninuclear polarizing polygon mode illustrating the urbanization sprawl over time to all directions. The greatest expansions are seen in NNE and SSE direction, mainly because of the topographic constraints. A trade-off between urbanization and sustainable development is under investigation by reclaiming barren soil at the mountain range to transfer farming areas from low altitude plains to make way for urban growth.
\end{abstract}

\section{Keywords}

Urbanization, Land Use Change, Landsat Archived Data, Yuxi, Hongta

District, China

\section{Introduction}

In the past three decades, an unprecedented urbanization has taken place globally [1], and urban expansion has affected ecosystem functioning and it associates with socioeconomic problems such as congestion, urban unemployment 
and lack of public services [2] [3] [4]. China is rapidly urbanizing, not just in Beijing and Shanghai, but in 3rd- and 4th-tier cities with a population of around one million. At present, urbanization in first-tier cities has been well-studied, but for medium-sized cities, which represent most of Chinese cities, it is relatively less understood. Yuxi city is a 4th-tier city and is known as the hometown of Yunnan tobacco, since its municipal heart, the Hongta district, center of tobacco producing and exporting, performs an evident contribution to GDP.

Characterizing urban expansion is the prerequisite to not only understand the urbanization process itself and its driving forces, but the ecological consequences and measures for optimal urban planning and management strategies.

\section{Objectives}

In this study, we aimed at the analysis and understanding the land transformation in Hongta District, the spatially explicit urban growth patterns and temporal change of landscape metrics.

\section{Data and Method}

\subsection{Study Area}

Yuxi is a prefecture-level city in the Yunnan province. Hongta District is the administrative center of Yuxi City, with an average elevation above $1800 \mathrm{~km}$. The coordinates of the Hongta District are presented in Table 1. A color infrared imagery (CIR) of the area is shown in Figure 1.

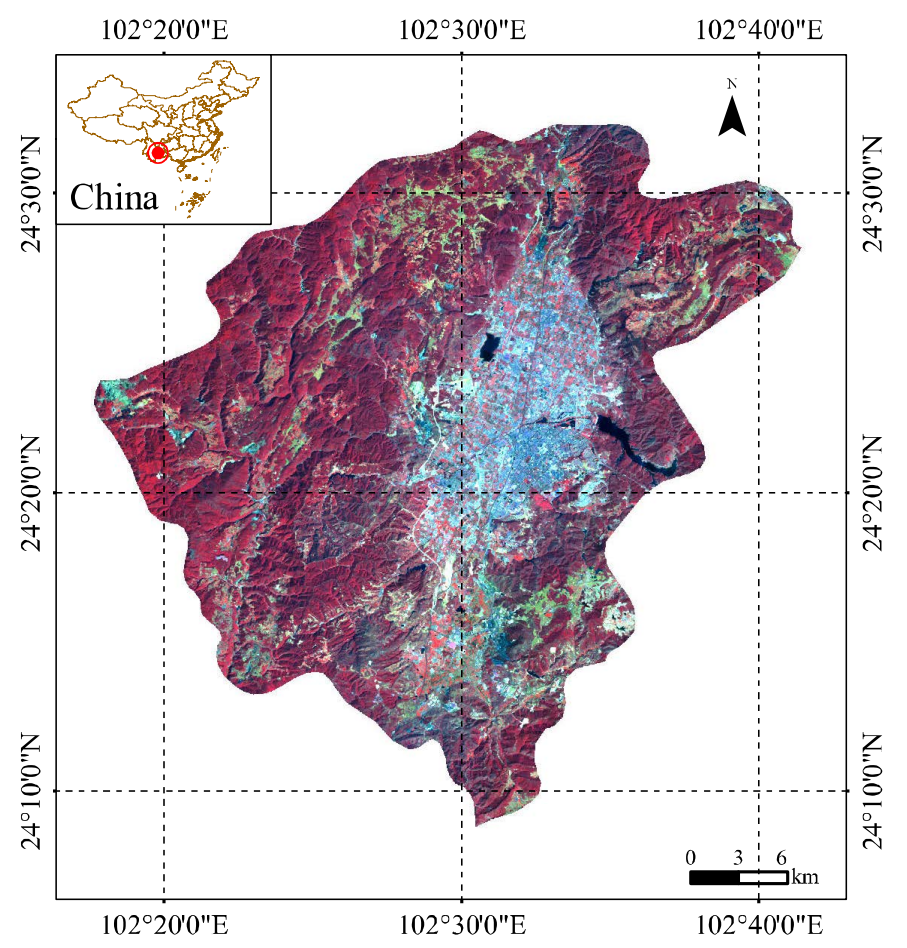

Figure 1. CIR image of Hongta District. Landsat bands 4, 3, 2 coded red, green, and blue. 
Table 1. Geographic setting of study area.

\begin{tabular}{cc}
\hline Latitude $(\mathrm{N})$ & $23^{\circ} 19^{\prime}-24^{\circ} 53^{\prime}$ \\
Longitude $(\mathrm{E})$ & $101^{\circ} 16^{\prime}-103^{\circ} 09^{\prime}$ \\
Elevation $(\mathrm{m})$ & $1505-2606$ \\
Area $\left(\mathrm{km}^{2}\right)$ & 963.3 \\
\hline
\end{tabular}

\subsection{Remote Sensing Data and Processing}

WeLandsat archived data have been obtained from the USGS website ((http://www.usgs.gov/) and are used to derive information about land use transformation between 1987 and 2017. Band combination, reprojection and image enhancement were completed by professional image processing software ENVI 5.3.1 and ArcGis 10.5. Five main land use types (water bodies, bare soils, farming areas, forests and sealed surfaces) were selected for classification. Because of seasonal phenological changes, farm lands and forests are divided into further sub-classes respectively. Training and testing samples are derived from Google Earth Pro, that covers the high-resolution images from Digital Globe and Airbus Defense. Several tests with different supervised classification methods based on equal training samples revealed the neural network method [5] [6] as most suitable for our approach. It showed the best overall accuracy and a Kappa coefficient up to $92 \%$ on the average, and thus, met the accuracy requirements of land cover change evaluation [7]. Besides the Landsat data, Aster DEM data with 1-degree spatial resolution were used for further analysis.

\subsection{Analyses on Sealed Surfaces (Urban Area)}

We chose three class-level landscape metrics to describe land-scape changes [8]: Total Area (TA), Percentage of Landscape (PLAND) and Patch Density (PD), the definitions are shown in Table 2.

All these metrics were examined through different directions. We divided the city into 16 sectors with 22.5-degree intervals (N, NNE, NE, NEE, E, EES, ES, ESS, S, SSW, SW, SWW, W, WWN, WN, WNN). The centroid of the sealed surfaces was assumed as the city center, which is defined as follow [9]:

$$
X=\sum_{t=1}^{m}\left(\sum_{i=1}^{n}\left(C_{t i} \times X_{t i}\right) / \sum_{i=1}^{n} C_{t i}\right) / m, \quad Y=\sum_{t=1}^{m}\left(\sum_{i=1}^{n}\left(C_{t i} \times Y_{t i}\right) / \sum_{i=1}^{n} C_{t i}\right) / m
$$

where $X$ and $Y$ are the average center coordinates in 11 years, $C_{t i}$ is the area of patch of $i$ during $t$ year, and $X_{t i}$ and $Y_{t i}$ are the centroid coordinates of patch of $i$ during $t$ year.

To quantify and compare the magnitude of urban expansion over different periods, the Annual Growth Rate $(A G R)$ index was calculated defined as follows:

$$
A G R=100 \% \times\left[\left(\frac{A_{\text {end }}}{A_{\text {start }}}\right)^{1 / d}-1\right]
$$

where $A G R$ is the annual growth rate, $A_{\text {start }}$ and $A_{\text {end }}$ are the extent of urban area at the start and end period respectively, and $d$ is the time span between the chosen start and end year. 
Table 2. Landscape metrics used in this study.

\begin{tabular}{cc}
\hline Acronym & Description \\
\hline TA $\left(\mathrm{km}^{2}\right)$ & The whole urban area of study zone \\
PLAND $(\%)$ & The percentage of total landscape \\
PD (n/100ha) & The number of patches of per 100 ha \\
\hline
\end{tabular}

\section{Results}

\subsection{Land Use Change Detection}

Indicated by Figure 2, the Hongta District has undergone a rapid urban expansion with an increase of sealed surfaces from $1.4 \%$ to $11.8 \%$ (a factor of 8.4!) over the past three decades. The average increasing rate of $7.2 \%$ is displayed by Table 3. Forests and farming areas, both underwent a slight reduce from $64.1 \%$ and $31 \%$ to $25.4 \%$ and $61.1 \%$ respectively. The barren lands diminished from $3.0 \%$ to $1.1 \%$. According to the transfer matrix, $23.8 \%$ of farms and $9.8 \%$ of bare lands transferred into sealed surfaces, and during the same time period $63.1 \%$ of bare lands transferred into farms.

Figure 3 and Figure 4 represent the distribution of temporal-spatial changes of the sealed surfaces and farm areas with a DEM as background. Figure 3 shows an obvious and continuous urban expansion. Specifically, Hongta District has experienced its urban land expanding converge on the low altitude and relatively flat terrain area according to the DEM distribution. Besides, the expansion grows almost along every direction from the original urban core, performing a uninuclear expansion pattern.

According to Figure 4, though a slightly instable change occured on the area of farm lands that might be due to the different days of year (DOY). The distribution change of farm areas (Figure 4) shows a trend toward decentralization, performing a dispersion from the low altitude plain to the mountainous region.

\subsection{Expansion Pattern of Sealed Surfaces (Urban Area)}

To further understand the detailed spatial patterns of the landscape of the city, we divided the landscape metrics in different directions and the results are shown in Figure 5. TA (Figure 5(a)) increased over time for all directions with the greatest expansions to NNE and SSE and the second greatest to N and S. The changing pattern of TA depicts a central symmetry especially for the north-south direction. PD (Figure 5(b)) increased first and then decreased for all directions during 1996 and 1999. Finally continued increasing after 2001 with largest PD changed from ES to SSW. PLAND (Figure 5(c)) also shows an insistent expansion versus time and mostly extends to the east sector.

\section{Discussion}

Hongta District has experienced dramatic urban expansion over the past three decades displaying a uninuclear expansion pattern with an increase of sealed 


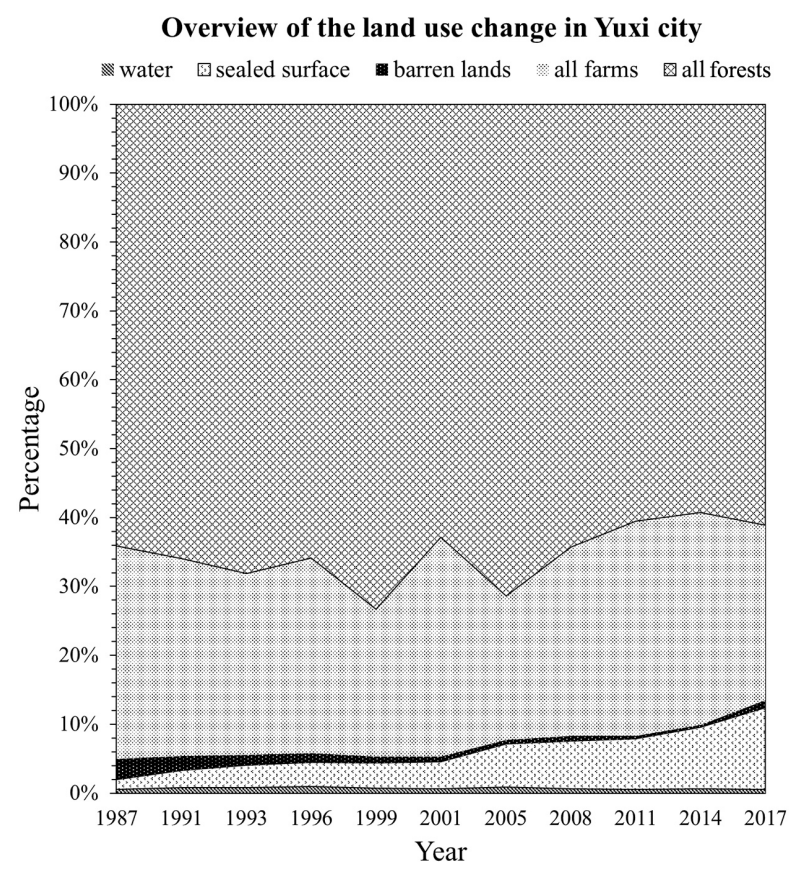

Figure 2. Land use change in Hongta District among three decades.

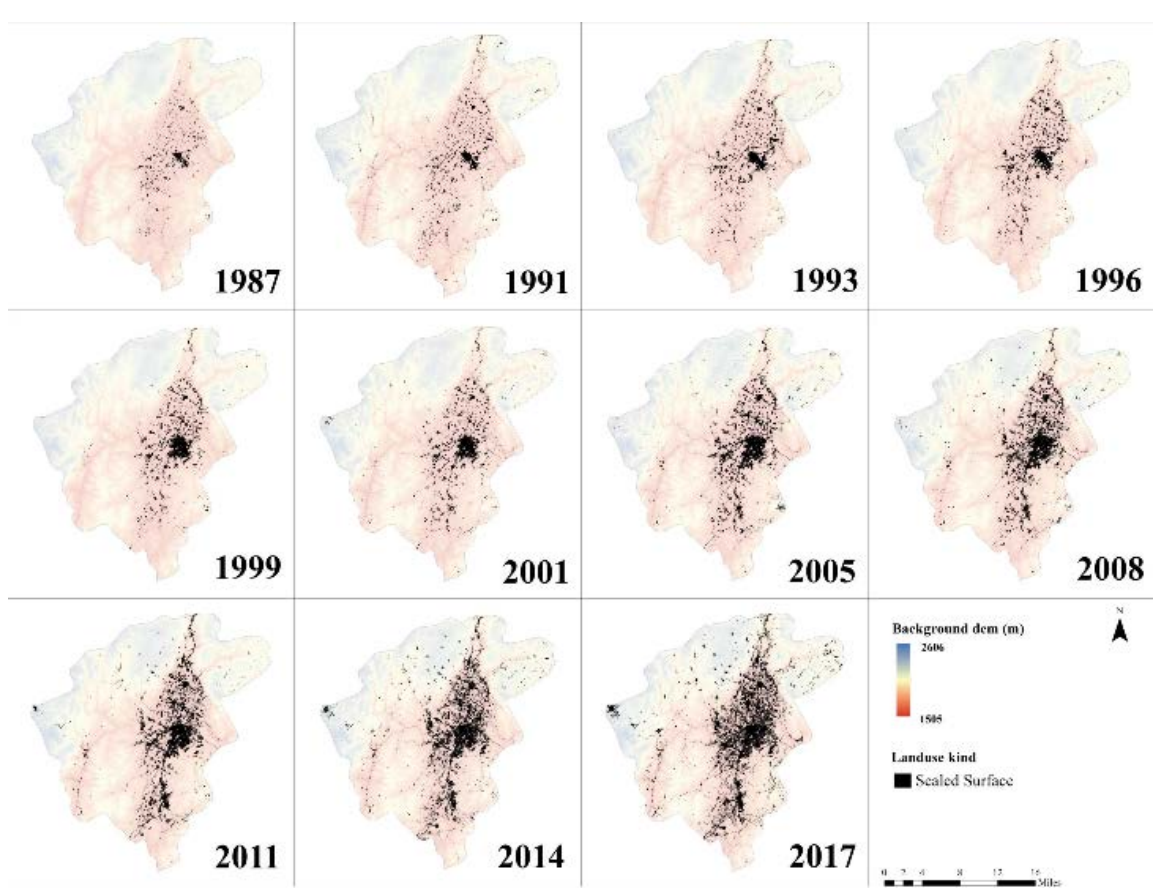

Figure 3. Temporal-spatial change of sealed surfaces (urban area).

Table 3. Annual growth rate (AGR) among 11 neighboring periods from 1987 to 2017.

\begin{tabular}{ccccccc}
\hline Time & $1987-1991$ & $1991-1993$ & $1993-1996$ & $1996-1999$ & $1999-2001$ & $2001-2005$ \\
\hline AGR & $15.9 \%$ & $14.8 \%$ & $2.1 \%$ & $1.7 \%$ & $3.0 \%$ & $12.4 \%$ \\
Time & $2005-2008$ & $2008-2011$ & $2011-2014$ & $2014-2017$ & Average & \\
AGR & $3.1 \%$ & $2.4 \%$ & $6.8 \%$ & $9.6 \%$ & $7.2 \%$ & \\
\hline
\end{tabular}




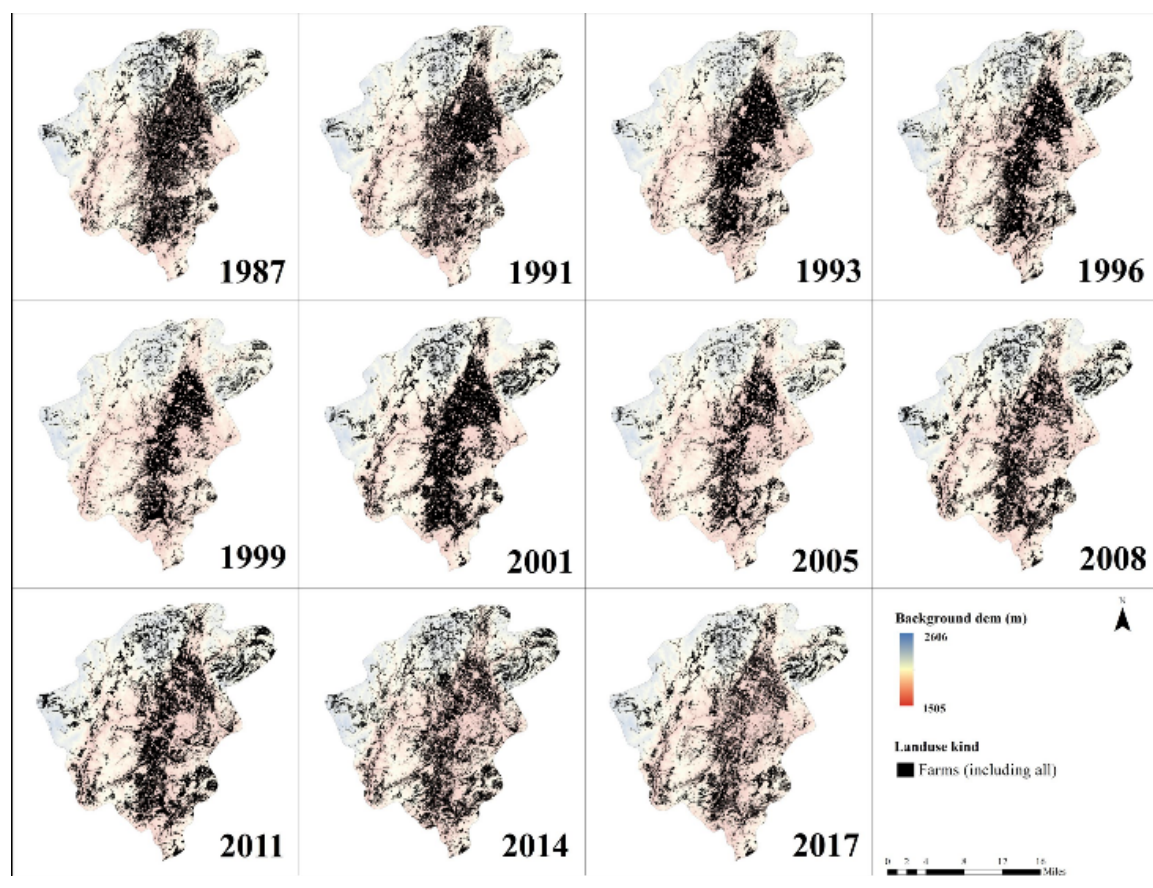

Figure 4. Temporal-spatial change of farm area.

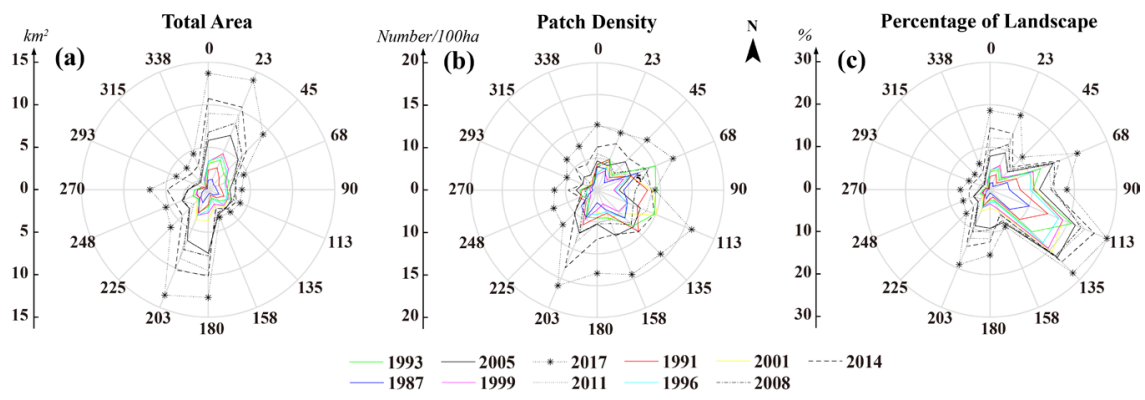

Figure 5. Landscape metrics for urban area in 16 sectors from 1987 to 2017: (a) Total area $\left(\mathrm{km}^{2}\right)$; (b) PD (per $100 \mathrm{ha}$ ) and (c) PLAND (\%).

surfaces by a factor of 8.4 and an AGR of 7.2\%. Physical factors such as elevation and slope are the basic constraints to urban expansion. Lower elevations and smaller slopes are generally associated with higher urban expansion. When urban expansion occupied most of the low plains, farming area had to move to other places, and $63.1 \%$ of bare lands are transferred into farm lands. According to AGR change during different periods, Hongta District experienced two most dramatic expansion periods in the years from 1987-1993 and 2001-200, that may be related to policies and/or special investments.

The effects of urban expansion in Hongta District on the landscape also vary with scale. At the regional scale, landscape fragmentation and irregularities increased in the early stage of urbanization, which is consistent with general observations. However, in 1996 and 1999 a decrease in landscape fragmentation and irregularity suggested that urban planning can adjust urban expansion types to avoid unrestricted acceleration, as urban expansion is not always monotonic 
but varies with space and time.

Although percentage of sealed surfaces increased for all three cities during the study period, there was a different increasing magnitude in various directions and at different distances within each city, indicating hotspots of urbanization changed with urban planning or policy in specific periods.

\section{Conclusion}

Urban expansion, that has affected ecosystem functioning and services at local to global scale, is projected to have aggravating impacts on the landscape in the future. A detailed study on spatiotemporal patterns of Yuxi City in Hongta District has been carried out over a relatively long timeframe using multi-temporal Landsat imagery and associated techniques and methods such as Geographic Information Systems and landscape analysis approaches. Results show the sealed surfaces (urban area) has expanded from $1.4 \%$ to $11.8 \%$, with annually increasing rate at $7.63 \%$, and forests, farm areas and barren lands all underwent a slight reduction during the past 30 years, whereby $23.8 \%$ of farms and $9.8 \%$ of bare lands transferred into sealed surfaces. As we focus on the urban expansion pattern, it presented a uninuclear polarizing polygon mode and the urbanization increased over time for all directions with the greatest expansion to NNE and SSE.

\section{Acknowledgements}

This research was conducted in the frame of the "Thousand Talents Program" financed by the Chinese Government and the University of Shandong. It did not receive any further specific grants from funding agencies in the public, commercial, or not-for-profit sectors.

\section{Conflicts of Interest}

The authors declare no conflicts of interest regarding the publication of this paper.

\section{References}

[1] Seto, K.C., Fragkias, M., GãNeralp, B. and Reilly, M.K. (2011) A Meta-Analysis of Global Urban Land Expansion. Plos One, 6, e23777. https://doi.org/10.1371/journal.pone.0023777

[2] Bolund, P. and Hunhammar, S. (1999) Ecosystem Services in Urban Areas. Ecological Economics, 29, 293-301. https://doi.org/10.1016/S0921-8009(99)00013-0

[3] Grimm, N.B., Faeth, S.H., Golubiewski, N.E., Redman, C.L., Wu, J., Bai, X., et al. (2008) Global Change and the Ecology of Cities. Science, 319, 756-760. https://doi.org/10.1126/science.1150195

[4] Mcdonnell, M.J. and Pickett, S.T.A. (1990) Ecosystem Structure and Function along Urban-Rural Gradients: An Unexploited Opportunity for Ecology. Ecology, 71, 1232-1237. https://doi.org/10.2307/1938259

[5] Richards, J.A. (1999) Remote Sensing Digital Image Analysis. Springer. 
https://doi.org/10.1007/978-3-662-03978-6

[6] Rumelhart, D.E. and Mcclelland, J.L. (1986) Parallel Distributed Processing. 1, MIT Press.

[7] Foody, G.M. (2002) Status of Land Cover Classification Accuracy Assessment. Remote Sensing of Environment, 80, 185-201.

https://doi.org/10.1016/S0034-4257(01)00295-4

[8] Mcgarigal, K. and Marks, B.J. (1995) FRAGSTATS: Spatial Analysis Program for Quantifying Landscape Structure. USDA Forest Service-General Technical Report PNW, 351. https://doi.org/10.2737/PNW-GTR-351

[9] JunHong, B., Hua, O., BaoShan, C., QingGai, W. and Hui, C. (2008) Changes in Landscape Pattern of Alpine Wetlands on the Zoige Plateau in the Past Four Decades. 28, 2245-2252. 